OPEN ACCESS

Edited by:

Beatrice Arosio,

University of Milan, Italy

Reviewed by:

Marcus Kiiti Borges, Independent Researcher, São Paulo,

Brazil

Gustavo Sevlever, Fundación Para la Lucha Contra las Enfermedades Neurológicas de la Infancia (FLENI), Argentina

*Correspondence: Yingchun Zhang yzhang94@uh.edu

Received: 09 December 2020 Accepted: 11 May 2021 Published: 10 June 2021

Citation:

Wu Z, Gao Y, Potter T, Benoit J, Shen J, Schulz PE, Zhang $Y$ and The Alzheimer's Disease Neuroimaging Initiative (2021) Interactions Between Aging and Alzheimer's Disease on Structural Brain Networks. Front. Aging Neurosci. 13:639795. doi: 10.3389/fnagi.2021.639795

\section{Interactions Between Aging and Alzheimer's Disease on Structural Brain Networks}

\author{
Zhanxiong Wu ${ }^{1}$, Yunyuan Gao ${ }^{2 *}$, Thomas Potter ${ }^{3}$, Julia Benoit ${ }^{4}$, Jian Shen ${ }^{5}$, Paul E. \\ Schulz ${ }^{6}$, Yingchun Zhang ${ }^{3 *}$ and The Alzheimer's Disease Neuroimaging Initiative \\ ${ }^{1}$ School of Electronic Information, Hangzhou Dianzi University, Hangzhou, China, ${ }^{2}$ Department of Intelligent Control and \\ Robotics Institute, College of Automation, Hangzhou Dianzi University, Hangzhou, China, ${ }^{3}$ Department of Biomedical \\ Engineering, University of Houston, Houston, TX, United States, ${ }^{4}$ Texas Institute for Measurement Evaluation and Statistics, \\ Department of Basic Vision Sciences, University of Houston, Houston, TX, United States, ${ }^{5}$ Neurosurgery Department, The \\ First Affiliated Hospital of Zhejiang University School of Medicine, Zhejiang University, Hangzhou, China, ${ }^{6}$ Department of \\ Neurology, The McGovern Medical School of UTHealth-Houston, Houston, TX, United States
}

Normative aging and Alzheimer's disease (AD) propagation alter anatomical connections among brain parcels. However, the interaction between the trajectories of age- and AD-linked alterations in the topology of the structural brain network is not well understood. In this study, diffusion-weighted magnetic resonance imaging (MRI) datasets of 139 subjects from the Alzheimer's Disease Neuroimaging Initiative (ADNI) database were used to document their structural brain networks. The 139 participants consist of 45 normal controls (NCs), 37 with early mild cognitive impairment (EMCl), 27 with late mild cognitive impairment (LMCl), and $30 \mathrm{AD}$ patients. All subjects were further divided into three subgroups based on their age (56-65, 66-75, and 71-85 years). After the structural connectivity networks were built using anatomically-constrained deterministic tractography, their global and nodal topological properties were estimated, including network efficiency, characteristic path length, transitivity, modularity coefficient, clustering coefficient, and betweenness. Statistical analyses were then performed on these metrics using linear regression, and one- and two-way ANOVA testing to examine group differences and interactions between aging and $A D$ propagation. No significant interactions were found between aging and $A D$ propagation in the global topological metrics (network efficiency, characteristic path length, transitivity, and modularity coefficient). However, nodal metrics (clustering coefficient and betweenness centrality) of some cortical parcels exhibited significant interactions between aging and $A D$ propagation, with affected parcels including left superior temporal, right pars triangularis, and right precentral. The results collectively confirm the age-related deterioration of structural networks in $\mathrm{MCl}$ and $\mathrm{AD}$ patients, providing novel insight into the cross effects of aging and $A D$ disorder on brain structural networks. Some early symptoms of $A D$ may also be due to age-associated anatomic vulnerability interacting with early anatomic changes associated with $A D$.

Keywords: Alzheimer's disease, structural network, nerve fiber tracking, diffusion-weighted magnetic resonance imaging, cognitive impairment 


\section{INTRODUCTION}

Increasing evidence suggests that both aging and Alzheimer's disease (AD) can cause deterioration in anatomical brain connections, which is then associated with a decline in cognitive abilities (Peters, 2002; Perl, 2010; Teipel et al., 2016). Normal aging can undermine white matter organization, as nerve fiber loss increases with age. This decrease in the connections between distinct brain parcels contributes to a disruption in the normal flow of information through cortical networks (Betzel et al., 2014; Zhao et al., 2015; Wu et al., 2020). As a neurodegenerative disorder that reduces synaptic transmission (Morabito et al., 2015), AD also causes a gradual breakdown in brain structural connectivity, eventually resulting in dementia (Voevodskaya et al., 2018; Dai et al., 2019; Wu et al., 2019). This disruption of structural connectivity between key functional subregions may ultimately explain the characteristic deficits found in $\mathrm{AD}$ patients (Yao et al., 2010; Fischer et al., 2015; delEtoile and Adeli, 2017; Li et al., 2020). These age- and AD-related alterations in white matter organization can profoundly affect topological features of the brain structural network and synergistically damage its integrity (Palop et al., 2006).

Diffusion-weighted imaging (DWI) has often been employed to assess cerebral white matter tracts (Tuch et al., 2003; Sinke et al., 2018; Innocenti et al., 2019; Sotiropoulos and Zalesky, 2019). Pioneering studies have then used graph theory to quantify the brain structural organization, reporting meaningful results on brain networks in normal aging and AD (Yao et al., 2010; Stawarczyk et al., 2012; Ghanbari et al., 2014; Zhao et al., 2015). In particular, alterations in the topology of brain structural networks and their corresponding metrics reflect the regional interactions as they evolve in both normal aging and in $\mathrm{AD}$ progression. When used to address normative aging, decreased network efficiency has been demonstrated in hub regions, limiting their capacity to communicate (Gong et al., 2009; Zhao et al., 2015). This is believed to result from degeneration in the white matter microstructure (demyelination, Wallerian degeneration, gliosis, severe fiber loss, etc.; Burzynska et al., 2010; Damoiseaux, 2017; Reishofer et al., 2018) and contributes to lifelong decline (van den Heuvel and Sporns, 2013; Betzel et al., 2014; Gollo et al., 2018). For mild cognitive impairment (MCI) and $\mathrm{AD}$, altered interregional correlations (particularly among the parahippocampal gyrus, medial temporal lobe, cingulum, fusiform, medial frontal lobe, and orbital frontal gyrus; Yao et al., 2010) lead to increased path lengths and decreased network efficiency (Lo et al., 2010; Fischer et al., 2015; delEtoile and Adeli, 2017), suggesting an impairment of structural networks in MCI and AD (He et al., 2008; Daianu et al., 2015; Raj et al., 2015). Especially, a structural $k$-core network analysis (examination of only nodes with a degree of $k$ or higher) was performed on normal controls (NCs) and $\mathrm{AD}$ patients to investigate brain network breakdown as AD progresses (Daianu et al., 2013). This study found that white matter integrity deteriorated with age and was able to distinguish early MCI-linked white matter alterations from those that occurred during normal aging. The fact that aging and cognitive impairment could separately affect brain networks highlights the unique effects that each has on brain network topology. The interaction of these effects, however, has not yet been thoroughly addressed.

Considering that age effects are not restricted to healthy individuals, it is likely that the age-related disruption of structural networks can exacerbate the cognitive decline in MCI and AD patients. It is, therefore, necessary to recognize the distinct effects of aging and impairment on the brain structural networks, and how these separate factors can interact within both healthy individuals and those with MCI and AD. At the present time, age-related alterations in the structural networks of MCI and $\mathrm{AD}$ patients have not been comprehensively explored. In this study, the data from 139 subjects, obtained from Alzheimer's Disease Neuroimaging Initiative (ADNI) database (Jack et al., 2008) and divided into three age subgroups (56-65, 66-75, and 71-85 years), were used to assess the deterioration of structure that occurs with age. We included $45 \mathrm{NCs}, 37$ early MCI (EMCI), 27 late MCI (LMCI), and $30 \mathrm{AD}$ patients. Statistical analysis focused on the cross effects between aging and $\mathrm{AD}$ progression on the topology of structural connectivity networks and investigated how the global and nodal topological metrics change with age, including network efficiency, characteristic path length, transitivity, modularity coefficient, clustering coefficient, and betweenness. From whole perspective, this study provides a complete view of $\mathrm{AD}$-related topological changes in brain structural connectomes over time.

\section{MATERIALS AND METHODS}

\section{Data}

We used the ADNI database (adni.loni.usc.edu), launched in 2003 as a public-private partnership and led by Principal Investigator Michael W. Weiner, MD. The primary goal of ADNI has been to test whether magnetic resonance imaging (MRI), positron emission tomography (PET), biomarkers, and clinical and neuropsychological assessment can be combined to measure the progression of MCI and early AD (Jack et al., 2008; Risacher et al., 2009; Petersen et al., 2010). In this study, 139 subjects aged from 56 to 85 years were selected from the ADNI database, including 45 NCs (32 females and 13 males), 37 EMCIs (18 females and 19 males), 27 LMCIs (11 females and 16 males), and $30 \mathrm{ADs}$ (13 females and 17 males). The criteria for the classification of the subjects was based on mini-mental state examination (MMSE) and global clinical dementia rating (CDR) scores (Aisen et al., 2010). Whole-brain Diffusion-weighted imagings (DWIs) were collected from four MRI centers using the: (1) Siemens 3T scanner (7 b0 images, 48 DWIs with $b=1,000 \mathrm{~s} / \mathrm{mm}^{2}$, slice thickness $=2 \mathrm{~mm}$, scanning sequence $=\mathrm{EP}$, echo time $=0.056 \mathrm{~s}$, repetition time $=7.2 \mathrm{~s}$, flip angle $\left.=90^{\circ}\right)$; $(2)$ the Siemens $3 \mathrm{~T}$ scanner (13 b0 images, 48 DWIs with $b=1,000 \mathrm{~s} / \mathrm{mm}^{2}$, slice thickness $=2 \mathrm{~mm}$, scanning sequence $=\mathrm{EP}$, echo time $=0.071 \mathrm{~s}$, repetition time $=3.4 \mathrm{~s}$, flip angle $\left.=90^{\circ}\right)$; $(3)$ the GE $3 \mathrm{~T}$ scanner (6 b0 images, 48 DWIs with $b=1,000 \mathrm{~s} / \mathrm{mm}^{2}$, slice thickness $=2 \mathrm{~mm}$, scanning sequence $=$ EP_SE, echo time $=0.0606 \mathrm{~s}$, repetition time $=7.8 \mathrm{~s}$, flip angle $=90^{\circ}$ ); and, the (4) Philips 3T scanner (1 b0 image, 32 DWIs with $b=1,000 \mathrm{~s} / \mathrm{mm}^{2}$, slice thickness $=2 \mathrm{~mm}$, scanning sequence $=\mathrm{SE}$, echo time $=0.099 \mathrm{~s}$, repetition time $=10.90 \mathrm{~s}$, flip 
angle $=90^{\circ}$; Daianu et al., 2013, 2015; Nir et al., 2015). ADNI data collection was performed after obtaining written informed consent from the participants. All procedures were in accordance with the ethical standards of the institutional and/or national research committee and with the 1964 Helsinki Declaration and its later amendments or comparable ethical standards. All DWI images were first denoised and corrected for eddy current and head movement distortions using MRtrix ${ }^{1}$ and $\mathrm{FSL}^{2}$ toolboxes. Then, DWI bias field correction was performed by estimating the bias field from b0 images. The whole flowchart of brain structural network construction by DWI is demonstrated in Figure 1.

Of note, there is still considerable controversy in the literature on the statistical analysis of structural network topology from multicenter DWI datasets (Tong et al., 2019). However, we suggest that topological characterization of structural networks would not significantly suffer from multicenter studies, as individual-based analysis of diffusion measures is not sensitive to the variability in MRI scanners (Tong et al., 2020). For the sake of reproducibility, the subject identifiers of each group are provided as Supplementary Materials.

\section{Structural Network Construction}

Brain structural networks can be represented as a graph, completely described by assigning a set of nodes and a set of edges with their corresponding weights (Hagmann et al., 2008; Garcés et al., 2016; Maier-Hein et al., 2017). In order to attain regional anatomical connectivity, the DKT template was applied to parcellate the whole brain into 62 subcortical regions (Potvin et al., 2017). Figure 1D demonstrates the DKT parcellation template, and Table 1 lists the indices of regions of interests (ROIs). This template was co-registered into DWI native space to define ROIs for each subject. The MRtrix tool ${ }^{1}$ was employed to reconstruct fiber tracks using deterministic tractography based on orientation distribution function (ODF) computed with constrained spherical deconvolution (CSD; Tournier et al., 2008). After fiber tracks were retrieved with the command "tckgen -act," spherical-deconvolution informed filtering of tractograms (SIFT) was employed to improve wholebrain streamlines reconstructions with the command "tcksift." Then, an inter-regional anatomical connectivity matrix was then obtained with "tck2connectome -symmetric-zero_diagonal," where the value of any element of the matrix is equal to the number of tracts originating in one region and terminating in (or passing to) another region. The number of fiber tracts between gray matter regions uncovered by MRtrix was determined from the data rather than defined a priori, and was therefore variable from individual to individual and from scan to scan (Bassett et al., 2011). Finally, the structural connectivity matrices were normalized into $[0,1]$ for topological characterization.

\section{Topological Characterization}

To characterize the underlying topological properties of brain structural networks, four commonly-used networklevel and two nodal topological metrics were computed for

\footnotetext{
${ }^{1}$ https://www.mrtrix.org

${ }^{2}$ https://fsl.fmrib.ox.ac.uk/
}

each subject: efficiency, characteristic path length, transitivity, modularity coefficient, clustering coefficient, and betweenness centrality. These metrics were directly retrieved from structural connectivity matrices using the Brain Connectivity Toolbox ${ }^{3}$ in MATLAB (The Mathworks, Inc., Natick, MA, USA; Rubinov and Sporns, 2010).

Network efficiency is a sensitive measure of network alterations that occur in aging and neurodegenerative disorders, which reflects the integration of information transfer within a given network. This effectively characterizes how well the information is communicated within the cerebral cortex and is expected to decrease with age (Gong et al., 2009). This metric is defined as:

$$
E_{\text {glob }}(G)=\frac{1}{N(N-1)} \sum_{i \neq j \in G} \frac{1}{L_{\mathrm{ij}}}
$$

where $L_{\mathrm{ij}}$ is the shortest path length between node $i$ and $j$ in structural connectivity graph G. $N$ denotes the number of nodes in the graph $G$.

The network characteristic path length is the average shortest path length between every pair of nodes in the network, which serves as a measure of overall network integration. This metric is inversely related to network efficiency (Cao et al., 2013) and quantifies the ability for information to be propagated in parallel. This metric was computed as:

$$
L(G)=\frac{1}{N(N-1)} \sum_{i \neq j \in G} L_{\mathrm{ij}}
$$

where $L_{\mathrm{ij}}$ is defined as the shortest path between node $i$ and node $j$.

Transitivity measures the probability that the adjacent vertices of a vertex are connected, which is closely related to the clustering coefficient of a graph, as both measure the relative frequency of triangles (Rubinov and Sporns, 2010).

$$
T(G)=\frac{3 \lambda(G)}{\tau(G)}
$$

where $\lambda(G)$ is the number of triangles in $G$, and $\tau(G)$ is total number of connected triples of nodes in $G$.

The optimal community structure is a subdivision of the network into nonoverlapping groups of nodes in a way that maximizes the number of within-group edges and minimizes the number of between-group edges. Modularity coefficient is a statistic that quantifies the degree to which the network may be subdivided into such clearly delineated groups. The modularity coefficient is defined as (Rubinov and Sporns, 2010):

$$
Q(G)=\frac{1}{2 m} \sum_{\mathrm{i}, \mathrm{j}}\left[w_{\mathrm{i}, \mathrm{j}}-\frac{k_{\mathrm{i}} k_{\mathrm{j}}}{2 m}\right] \delta\left(c_{\mathrm{i}}, c_{\mathrm{j}}\right)
$$

where $w_{\mathrm{i}, \mathrm{j}}$ is the connection weight between node $i$ and $j . k_{\mathrm{i}}$ and $k_{\mathrm{j}}$ are the sums of the weights of the edges attached to nodes $i$ and $j$, respectively. $m$ is the total link weight in the network overall. $\delta\left(c_{\mathrm{i}}, c_{\mathrm{j}}\right)$ is 1 when nodes $i$ and $j$ are assigned to the same

\footnotetext{
$\overline{3}$ http://www.brain-connectivity-toolbox.net/
} 
A

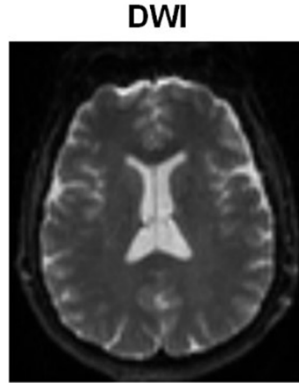

G
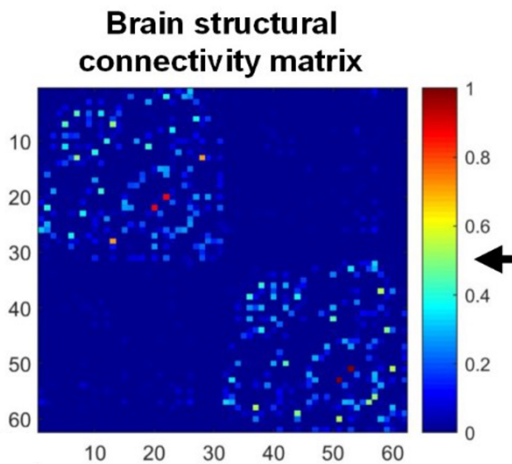
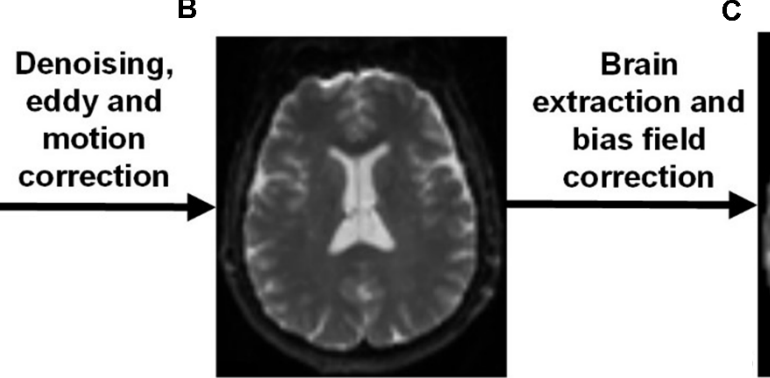

C
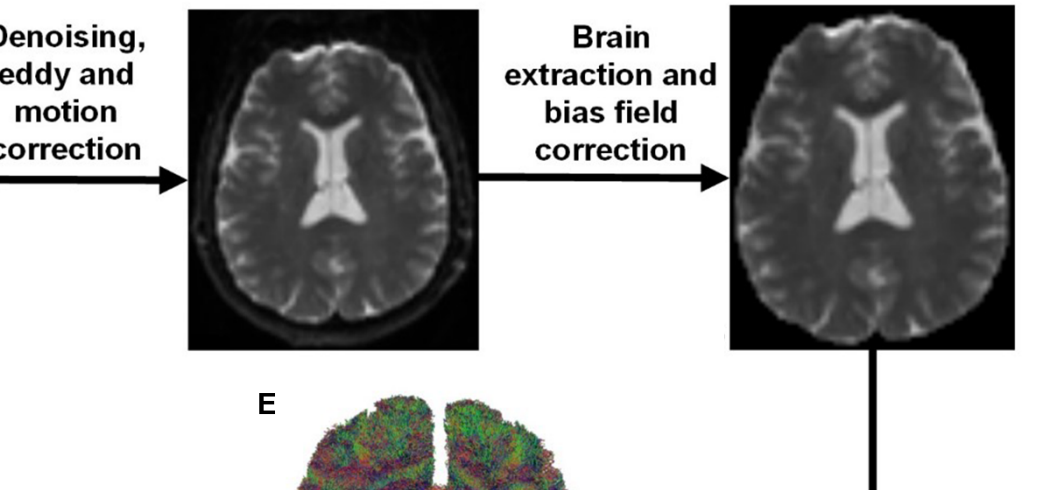

Fiber tracking

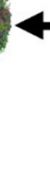

$\mathbf{F}$
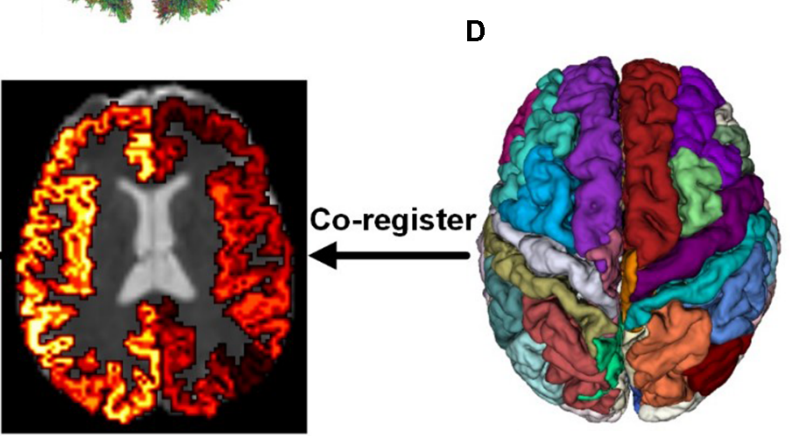

FIGURE 1 | Flowchart of brain structural network construction from diffusion-weighted imaging (DWI). Whole-brain models were parcellated into 62 different parcels according to the DKT template. (A) DWI. (B) After DWI was denoised, eddy and motion correction were performed. (C) Brain extraction and bias field correction. (D) DKT parcellation atlas. (E) White matter fibers reconstructed with anatomically-constrained tractography (Smith et al., 2012). (F) DKT atlas was co-registered into DWI native space. (G) Brain structural connectivity matrix was built by assigning fibers to each parcel.

TABLE 1 | Indexes of ROls used to construct structural networks (Tzourio-Mazoyer et al., 2002).

\begin{tabular}{|c|c|c|c|c|}
\hline Region & Region & Region & Region & Region \\
\hline 1 Left caudal anterior cingulate & 14 Left parahippocampal & 27 Left superior parietal & 40 Right lateral occipital & 53 Right precentral \\
\hline 2 Left caudal middle frontal & 15 Left paracentral & $\mathbf{2 8}$ Left superior temporal & 41 Right lateral orbitofrontal & 54 Right precuneus \\
\hline 3 Left cuneus & 16 Left pars opercularis & 29 Left supramarginal & 42 Right lingual & $\begin{array}{l}55 \text { Right rostral anterior } \\
\text { cingulate }\end{array}$ \\
\hline 4 Left entorhinal & 17 Left pars orbitalis & $\mathbf{3 0}$ Left transverse temporal & 43 Right medial orbitofrontal & $\begin{array}{l}56 \text { Right rostral middle } \\
\text { frontal }\end{array}$ \\
\hline 5 Left fusiform & 18 Left pars triangularis & 31 Left insula & 44 Right middle temporal & 57 Right superior frontal \\
\hline 6 Left inferior parietal & 19 Left pericalcarine & 32 Right caudal anterior cingulate & 45 Right parahippocampal & 58 Right superior parietal \\
\hline 7 Left inferior temporal & 20 Left postcentral & 33 Right caudal middle frontal & 46 Right paracentral & 59 Right superior temporal \\
\hline 8 Left isthmus cingulate & 21 Left posterior cingulate & 34 Right cuneus & 47 Right pars opercularis & 60 Right supramarginal \\
\hline 9 Left lateral occipital & 22 Left precentral & 35 Right entorhinal & 48 Right pars orbitalis & 61 Right transverse tempora \\
\hline 10 Left lateral orbitofrontal & 23 Left precuneus & 36 Right fusiform & 49 Right pars triangularis & 62 Right insula \\
\hline 11 Left lingual & $\mathbf{2 4}$ Left rostral anterior cingulate & 37 Right inferior parietal & 50 Right pericalcarine & \\
\hline 12 Left medial orbitofrontal & 25 Left rostral middle frontal & 38 Right inferior temporal & 51 Right postcentral & \\
\hline 13 Left middle temporal & 26 Left superior frontal & 39 Right isthmus cingulate & 52 Right posterior cingulate & \\
\hline
\end{tabular}

module and 0 otherwise. Larger $Q$ values are indicative of a highly modular network organization, while lower $\mathrm{Q}$ values indicate a more uniform network structure.
To assess the effect of aging and AD progression on local brain regions, node clustering coefficient and betweenness centrality were estimated for each group. The weighted clustering 
coefficient is the average intensity of all triangles associated with each node, which indicates the extent of local interconnectivity or cliquishness in a network (Daianu et al., 2013; Otte et al., 2015).

$$
c_{\mathrm{i}}=\frac{2}{k_{\mathrm{i}}\left(k_{\mathrm{i}}-1\right)} \sum_{\mathrm{j}, \mathrm{k}}\left(w_{\mathrm{i}, \mathrm{j}} w_{\mathrm{j}, \mathrm{k}} w_{\mathrm{k}, \mathrm{i}}\right)^{1 / 3}
$$

where $k_{\mathrm{i}}$ is the degree of node $i$, and $w$ denotes the structural connection weight.

Node betweenness centrality is the number of shortest paths that pass through a node (Equation 5). High betweenness centrality values indicate more passages traversing a node. In this work, betweenness centrality was normalized to the range $[0,1]$ as betweenness/[(N-1)(N-2)] (Rubinov and Sporns, 2010).

$$
b_{\mathrm{i}}=\sum_{h \neq j, h \neq i, j \neq i} \frac{p_{\mathrm{hj}}(i)}{p_{\mathrm{hj}}}
$$

where $p_{\text {hj }}$ is the number of shortest paths between nodes $h$ and $j$, and $p_{\text {hj }}(i)$ is the number of shortest paths between $h$ and $j$ that pass through the node $i$.

\section{Statistical Analysis}

The objective of this study is to assess the interactive effects of aging and $\mathrm{AD}$ progression on topological properties of the brain structural network. After the gender covariate was regressed out, linear regression and ANOVAs were adopted for statistical analysis and performed. In order to estimate the changing trajectories of topological measures over age, linear regression was separately performed on each global metric in the NC, EMCI, LMCI, and AD groups, respectively. To test whether the network-level topology of structural networks was significantly different over age and across NC, EMCI, LMCI, and AD groups, group-wise comparisons of network-level topological measures were performed using one-way ANOVA tests. Finally, to characterize the interaction between aging and $\mathrm{AD}$ progression on network-level and nodal topological properties, two-way ANOVA tests with the two factors of age and $\mathrm{AD}$ propagation stage were employed to identify group-wise differences. The factor of age consists of three levels: 56-65 years, 66-75 years, and 76-85 years. And the factor of the AD stage includes four levels: NC, EMCI, LMCI, and AD. A significance level of $p$-value $<0.05$ (uncorrected) was used for ANOVA tests.

\section{RESULTS}

\section{Linear Regression on Network-Level Topological Metrics}

Linear regression was performed on the global topological metrics (network efficiency, characteristic path length, transitivity, and modularity coefficient) to examine whether, over age, the structural networks of MCI and AD patients exhibited similar deterioration patterns. Figure 2 shows the results, which indicate that the characteristic path lengths (Slope: 0.21, 0.12, $0.18,0.05)$ of the NC, EMCI, LMCI, and AD groups increased with age, while the metric of efficiency (Slope: $-0.0004,-0.0002$, $-0.0006,-0.0002)$ decreased. However, except for the EMCI group, the transitivity of the $\mathrm{NC}, \mathrm{LMCI}$, and $\mathrm{AD}$ groups were nearly unchanged. While modularity coefficients (Slope: 0.0010, $0.0008,0.0002)$ of NC, EMCI, LMCI groups increase with age, the coefficient of the $\mathrm{AD}$ group remained unchanged. $\mathrm{R}$-square values of the linear regression were present on the left corner of each subgraph. Overall, linear regression results indicated that the integrity of the structural networks of $\mathrm{NC}, \mathrm{MCI}$, and $\mathrm{AD}$ individuals all roughly deteriorated with age. However, lesser age-related effects were found in the metrics of the AD group.

\section{ANOVA Tests on Topological Measures}

Differences in the global topological measures between the three age subgroups (56-65, 66-75, and 71-85 years) were assessed using one-way ANOVA tests. Figure 3 demonstrates the comparison results, and the asterisk sign $(*)$ indicates that $p$-value $<0.05$ (uncorrected). For the NC group, differences between age subgroups in network efficiency and characteristic path were statistically significant ( $p$-value $=0.0116$ and $p$-value $=0.0134$, respectively). For EMCI, differences in efficiency, characteristic path and clustering coefficient were significant $(p$-value $=0.0467, p$-value $=0.0256$ and $p$ value $=0.0069$, respectively). For LMCI subjects, differences between age groups in efficiency and clustering coefficient were significant ( $p$-value $=0.0211$ and $p$-value $=0.0315$, respectively). No metrics significantly differentiated the two age subgroups within the AD group.

Additionally, to detect group-wise differences among NC, EMCI, LMCI, and AD subjects, one-way ANOVA tests were also carried out. Results are shown in Figure 4, and pairwise groups that exhibited significant differences were identified and marked with an asterisk sign $\left(^{*}\right)$, indicating that $p$ value $<0.05$ (uncorrected). For the three age groups, efficiency and characteristic paths do not significantly distinguish the NC, EMCI, LMCI, and AD groups. However, for the three age groups, significant differences were only found in the metric of the modularity coefficient. Interestingly, a significant difference between the NCs and LMCI groups was only found in the 56-65 years group. This may be attributed to individual variability. In summary, while the modularity coefficient was most sensitive to $\mathrm{AD}$ propagation across 56-75 years, no significant difference was identified in terms of this metric among NC, LMCI, and AD subjects in the 76-85 years group.

Two-way ANOVA tests were also separately performed on the global topological metrics, and the combined changing trajectories of mean values of these metrics are shown in Figure 5. In summary, no significant interactions were found between aging and $\mathrm{AD}$ propagation in terms of these network-level metrics.

To reveal the interactive effect of aging and $\mathrm{AD}$ progression on local topological properties, nodal clustering coefficient and betweenness centrality were estimated for each subject. Using two-way ANOVA tests, it was found that multiple regions, including the left lateral occipital (9), left postcentral (20), right caudal anterior cingulate (32), right inferior parietal (37), right rostral anterior cingulate (55), and right superior frontal (57) exhibited significant differences in terms of clustering coefficient over age (Table 2 and Figure 6). Moreover, significant differences were found only in the parcel of right insula 

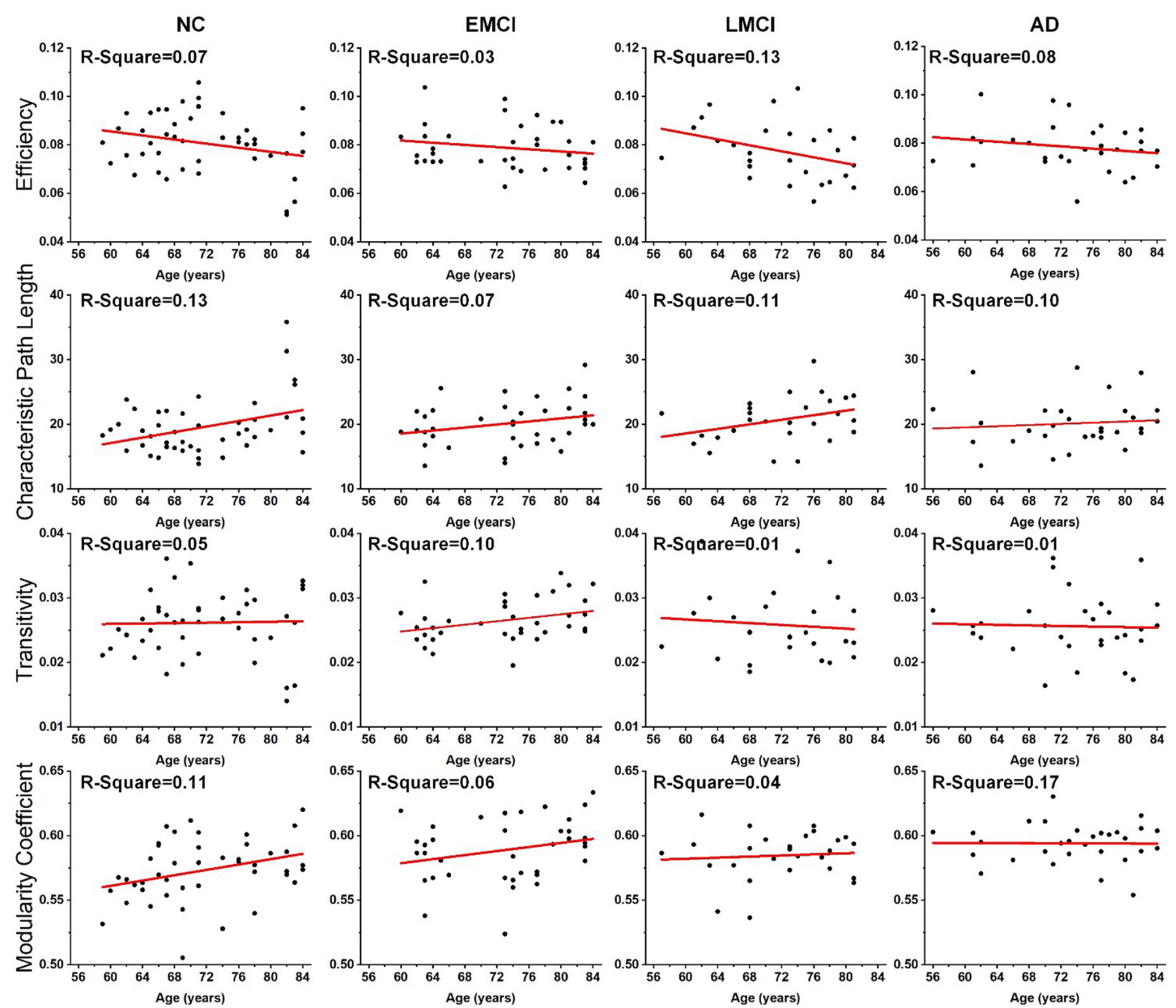

FIGURE 2 | Age effects on the global topological properties of structural networks, including global efficiency, characteristic path length, transitivity, and modularity coefficient. $R$-square value is present on the left corner of each subgraph. The fitted lines are shown in red, and the black dots represent the metric values of each subject.

(62) across AD propagation stages age (Table 2 and Figure 6). Regional betweenness centrality values in the left entorhinal (4), left fusiform (5), left middle temporal (13), left posterior cingulate (21), right lingual (42), right precentral (53), right rostral anterior cingulate (55), and right superior frontal (57), showed significant differences across age subgroups (Table 2 and Figure 6). In addition, the left rostral anterior cingulate (24) and right insula (62) parcels were identified to have significant differences across $\mathrm{AD}$ stages in terms of betweenness (Table 2 and Figure 6). Finally, for clustering coefficient, the cortical parcels of right pars triangularis (49) and right precentral (53) exhibit significant interaction between aging and $\mathrm{AD}$ propagation stages (Table 2 and Figure 7A). For the metric of betweenness, significant interactions between aging and $\mathrm{AD}$ stages were found in the left superior temporal (28) and right pars triangularis (49) (Table 2 and Figure 7A). The cortical parcels that exhibited significant groupwise differences and interactions are summarized in Table 2, and the corresponding positions of these parcels are displayed in Figures 6, 7A.

\section{DISCUSSION}

Cortical connectivity can be seen to reduce with age and $\mathrm{AD}$ progression, leading to significant deficits in topological properties of the structural network. These topological metrics provide valuable insights into the deteriorating neurological processes underlying aging and $\mathrm{AD}$ progression, offering a unique way to evaluate the impairment of anatomical connectivity patterns. In this study, we constructed brain structural networks of NC, EMCI, LMCI, and AD subjects by calculating fiber bundle numbers between pairs of gray matter parcels and investigating the cross effects of aging and $\mathrm{AD}$ progression on network-level and nodal structural topography. The results confirm that normal aging and $\mathrm{AD}$ propagation could 


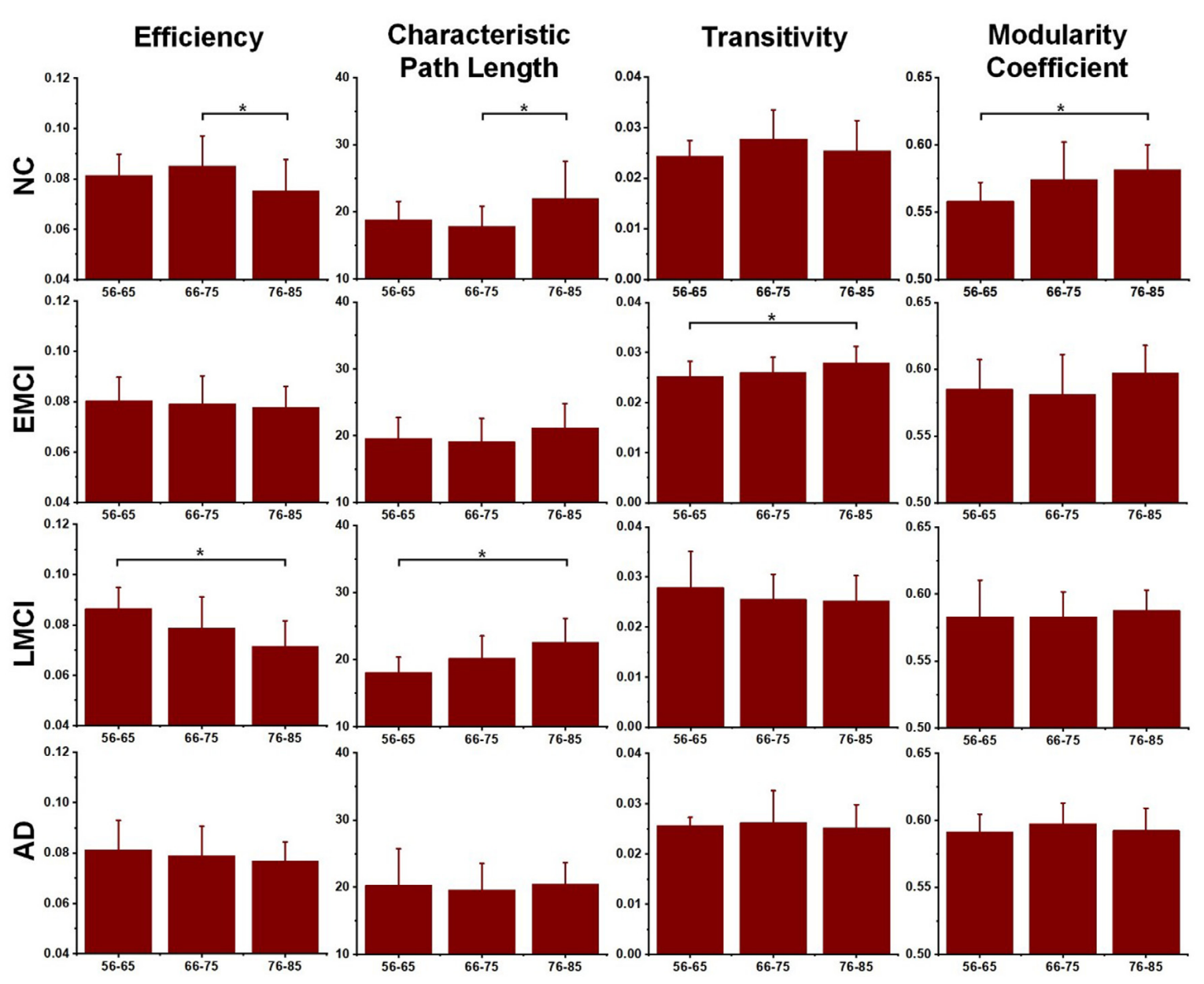

Age subgroups: $56-65,66-75$, and $76-85$ years.

FIGURE 3 | Differences between age subgroups (56-65, 66-75, and 76-85 years) in network efficiency, characteristic path length, transitivity, and modularity coefficient. Group differences were estimated using one-way ANOVA, and the asterisk sign $\left(^{*}\right)$ indicates that $p$-value $<0.05$ (uncorrected).

both affect the integrity of brain structural networks, and indicate that the network-level metrics of AD structural networks were relatively more deteriorated than those of NCs. Overall, however, more significant age-related differences were indicated in healthy controls than in $\mathrm{AD}$ patients.

Several recent studies based on DWI have demonstrated that the efficiency of structural networks decreases during normal aging due to neuronal shrinkage, loss of axon fibers, and whiter matter degeneration (Gong et al., 2009; Zhao et al., 2015; Sheffield et al., 2019). To reveal age-related degeneration in the white matter microstructure of NCs, MCI, and $\mathrm{AD}$, this study performed linear regression on each of the global topological metrics, separately. Results provided new insight into the age-related changes in brain structural networks of healthy, MCI, and $\mathrm{AD}$ individuals, which are crucial for understanding how age affects the structural connectome of AD disorders. For all groups, network efficiency decreased with increasing age while characteristic path length increased. This is in accordance with previous studies (Meunier et al.,
2009; Betzel et al., 2014; Fischer et al., 2015; Zhao et al., 2015). As shown in Figure 2, the deteriorated network-level topological properties of brain structural networks found in this study may provide the underlying substrate for the functional decline observed in aging individuals. In terms of the metrics of efficiency, characteristic path length, and modularity coefficient, the age-related deterioration in structural networks of $\mathrm{AD}$ patients is less significant than for healthy and older adults with MCI. We may infer that the anatomical connectivity breakdown caused by $\mathrm{AD}$ weakens the detrimental effect of aging on brain structural networks. Additionally, no significant age-related differences were identified in the $\mathrm{AD}$ subgroup (Figure 3), weighing against the hypothesis that aging leads to a vulnerability to the spread of $\mathrm{AD}$.

$\mathrm{AD}$ progression can be characterized by a loss of connected areas in terms of global topological measures including network efficiency, characteristic path length, transitivity, and modularity coefficient. Much evidence from previous studies supports the interpretation of $\mathrm{AD}$ as a disconnection syndrome 


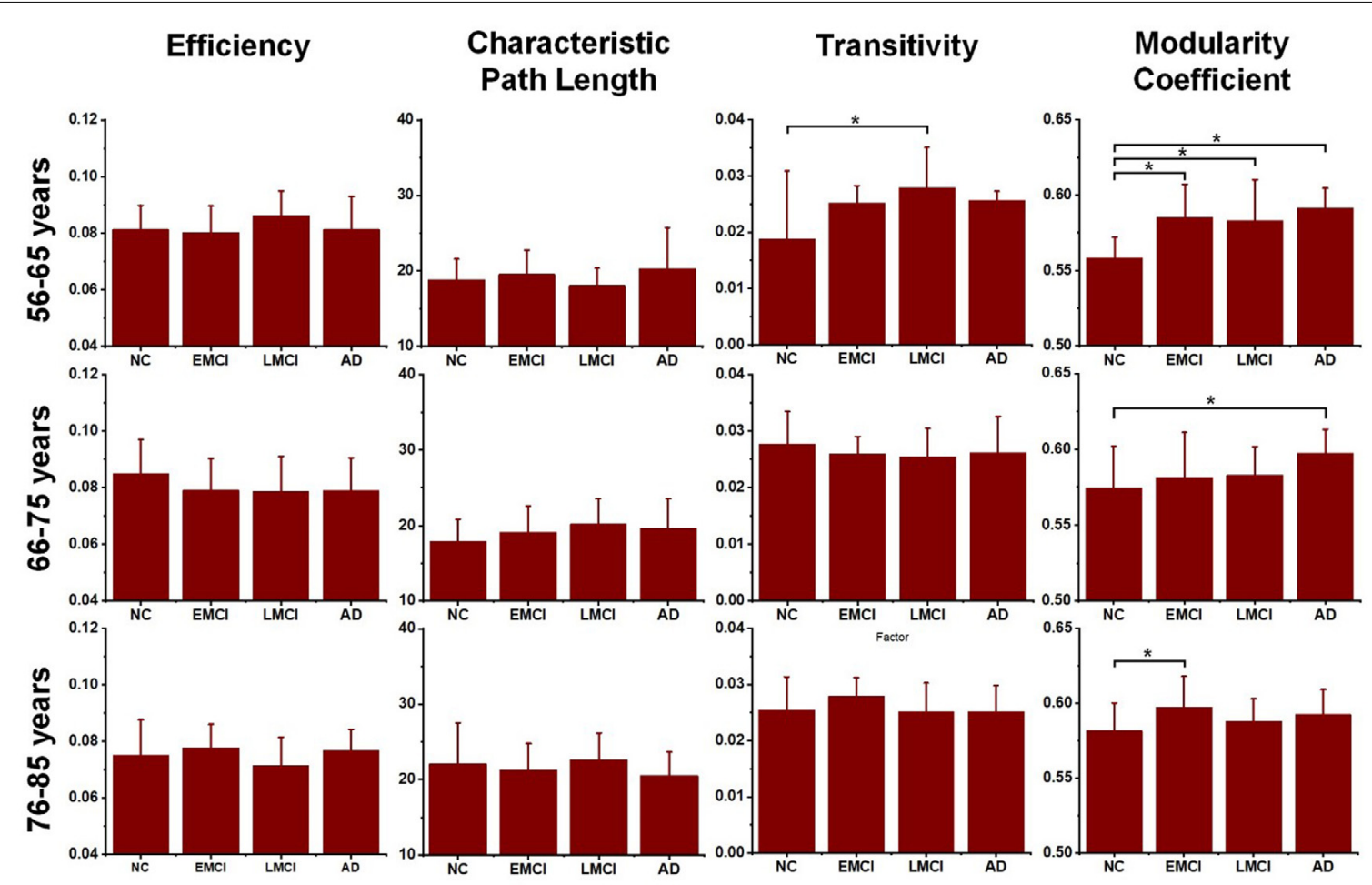

AD progression stages: NC, EMCI, LMCl, and AD.

FIGURE 4 | Differences across the normal control (NC), early mild cognitive impairment (EMCl), late mild cognitive impairment (LMCl), and Alzheimer's disease (AD) groups were estimated using one-way ANOVA tests in terms of network efficiency, characteristic path length, transitivity, and modularity coefficient. The asterisk sign $\left({ }^{*}\right)$ indicates that the $p$-value was $<0.05$ (uncorrected).

(Fischer et al., 2015; Morabito et al., 2015; Guo et al., 2016). To further reveal how AD propagation affects structural networks, one-way ANOVA tests were employed to explore groupwise differences across the NC, EMCI, LMCI, and AD groups in each age subgroup (56-65, 66-75, and 76-85 years). Figure 4 demonstrates that in the 56-65- and 66-75-years age groups, there were significant differences between NCs and ADs in terms of modularity coefficient. For the 75-85 years group, however, no significant difference in modularity coefficient was detected, indicating that aging and $\mathrm{AD}$ both lead to intermodule disconnection in brain structural networks. Interestingly, as shown in Figure 4, the other global metrics (network efficiency, characteristic path length, and transitivity) did not exhibit significant differences between $\mathrm{NC}$ and $\mathrm{AD}$ subjects in the three age groups ( $p$-value $>0.05$ ). The reasons why there is no difference between the groups regarding efficiency and path length are manifold. This work is based on cross-sectional ADNI data. There are individual variabilities in brain structural networks. The progression stages of $\mathrm{AD}$ are generally defined by MMSE and CDR test scores. At present, it's unclear if the subjects with the same test score share the same brain structural networks. Another hypothesis could be that neural plasticity would alter structural connectivity during AD progression. Some subjects may have better neural plasticity than others. Hence, their connectivity could be better or worse than predicted.
These one-way ANOVA tests indicate that the AD subjects are associated with greater structural connectivity deterioration in younger adults, while cognitive impairments have relatively lesser effects on older adults. Age-related alterations of wholebrain white matter network properties of $\mathrm{AD}$ patients were not detectable. However, the underlying neurophysiological reasons may be worthy of further study.

To comprehensively reveal the interaction between aging and $\mathrm{AD}$ progression on brain structural networks, using two-way ANOVA tests, the cross effect of aging and AD progression on local topological properties has been assessed in terms of node clustering coefficients and betweenness centrality (Table 2, Figures 6, 7). The results indicate that aging and $\mathrm{AD}$ progression interactively and significantly affect some local regions, including the left superior temporal, right pars triangularis, and right precentral. This may occur due to broken anatomical connections between these cortical subregions and others, which were interactively affected by aging and $\mathrm{AD}$ progression. The three parcels are related to language understanding and motor movement (Foundas et al., 1996; Yousry et al., 1997; Aeby et al., 2013), and these cognitive functions both gradually deteriorate with age and AD progression. Previous studies have found that subjects with $\mathrm{MCI}$ and $\mathrm{AD}$ have a significant reduction in structural connectivity in the superior temporal lobe, medial temporal 



FIGURE 5 | Combined changing trajectories of the four network-level topological metrics (efficiency, characteristic path length, transitivity, and modularity coefficient). No significant interactions were found between aging and AD propagation at the level of $p$-value $<0.05$ (uncorrected).

TABLE 2 | The cortical subregions with significant differences were identified in terms of clustering coefficient and betweenness centrality.

\begin{tabular}{|c|c|c|c|}
\hline Nodal metrics & $\begin{array}{l}\text { The population means of Age are } \\
\text { significantly different }(p<0.5)\end{array}$ & $\begin{array}{l}\text { The population means of Stage are } \\
\text { significantly different }(p<0.5)\end{array}$ & $\begin{array}{l}\text { The interaction between Age and } \\
\text { Stage is significant }(p<0.5)\end{array}$ \\
\hline $\begin{array}{l}\text { Clustering } \\
\text { Coefficient }\end{array}$ & $\begin{array}{l}\text { left lateral occipital (9), } \\
\text { left postcentral (20), } \\
\text { right caudal anterior cingulate (32), } \\
\text { right inferior parietal (37), } \\
\text { right rostral anterior cingulate (55), } \\
\text { right superior frontal (57) }\end{array}$ & right insula (62) & $\begin{array}{l}\text { right pars triangularis (49), } \\
\text { right precentral (53) }\end{array}$ \\
\hline Betweenness & $\begin{array}{l}\text { left entorhinal (4), } \\
\text { left fusiform (5), } \\
\text { left middle temporal (13), } \\
\text { left posterior cingulate (21), } \\
\text { right lingual (42), } \\
\text { right precentral (53), } \\
\text { right rostral anterior cingulate (55), } \\
\text { right superior frontal (57) }\end{array}$ & $\begin{array}{l}\text { left rostral anterior cingulate (24), } \\
\text { right insula (62) }\end{array}$ & $\begin{array}{l}\text { left superior temporal }(28) \text {, } \\
\text { right pars triangularis }(49)\end{array}$ \\
\hline
\end{tabular}

The listed brain parcels in this table were visualized on Montreal Neurologic Institute 152 (MNI152) brain images, as shown in Figures 6, 7A. The numbers in parentheses were the indexes of cortical parcels (Table 1). The p-values were not corrected.

lobe, inferior parietal areas, and lingual gyri (Bell-McGinty et al., 2005; Yao et al., 2010; Zhao et al., 2015). Age-related structural network studies also revealed that regional efficiency reduced in the parietal and occipital lobes with age (Gong et al., 2009; Burzynska et al., 2010; Zhao et al., 2015). To some extent, our result is in accordance with these prior studies. Specifically, no significant group-wise difference or interaction was found in the occipital area and hippocampus subregions (14 and 45) in this study. A possible reason for this may be the choices of whole-brain parcellation atlas and nodal metrics. Additionally, as the most serious hippocampal pathology may be already present when the diagnosis of MCI or AD was made, hippocampal connections could not have much additional deteriorations over time. The present results are, to some extent, consistent with these studies: cognitive function deficits could be due to abnormalities in the connectivity between these brain areas. This regionspecific topological analysis provides insight into the aberrant topological patterns induced by interaction between aging and AD propagation. 
A

Left postcentral (20)

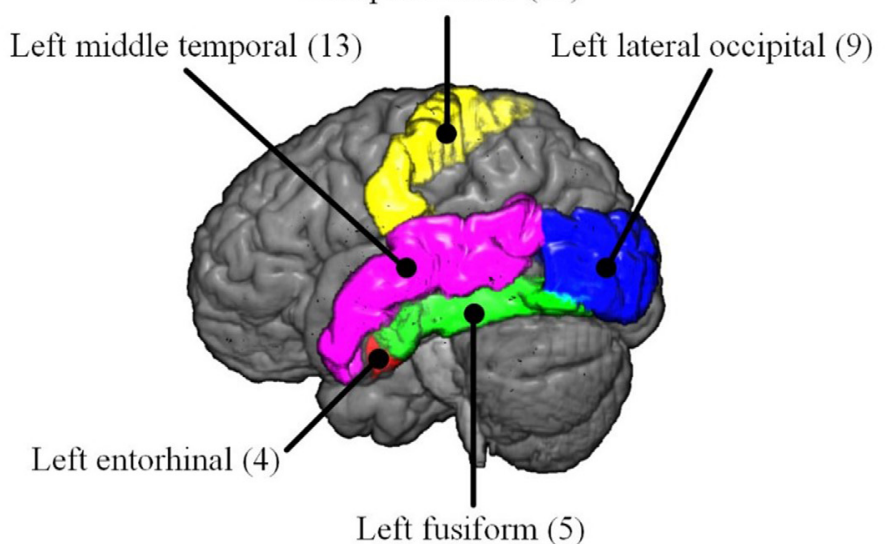

B

Right inferior parietal (37) Right precentral (53)

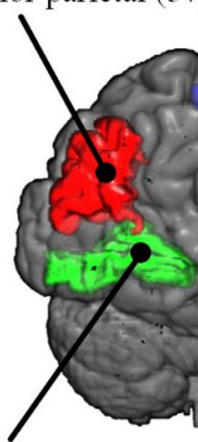

Right lingual (42)
Right insula (62)
Left posterior cingulate (21)

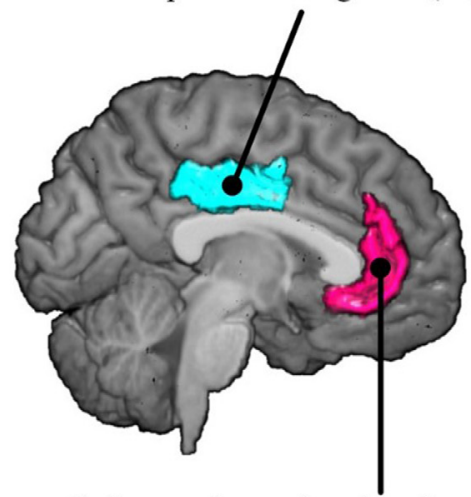

Left rostral anterior cingulate (24)

Right caudal anterior cingulate (32)



Right rostral anterior cingulate (55)

FIGURE 6 | The cortical parcels listed in Table 2 are visualized on MNI152 brain images. The numbers in parentheses are the indexes of parcels (Table 1). (A) Left hemisphere. (B) Right hemisphere.

Several methodological issues about this study should be addressed. First, the DKT atlas was used to parcellate the whole cortex. When different parcellation schemes are used to define network nodes, topological metrics may be different ( $\mathrm{Wu}$ et al., 2019). Second, the edges of the white matter networks were reconstructed by deterministic tractography based on CSD. Future studies should employ more advanced tractography techniques, such as probabilistic tractography to define the network edge weights (Sotiropoulos and Zalesky, 2019). Third, to ascertain the real structural networks as accurately as possible, this study included as many subjects as were available from each group in the ADNI database, which made the sample sizes of each group inconsistent. Fourth, as the DWI datasets are collected from multiple MRI centers, network consistency still needs to be confirmed. For different patients, $\mathrm{AD}$ onsets may start in distinct brain areas (Ossenkoppele et al., 2020), and this may influence the statistical analysis of local topological characterization. Finally, as the cause for white matter hyperintensities remain uncertain (Merio, 2019), we did not consider this factor in the statistical tests. Interaction across aging, $\mathrm{AD}$ progression, and neural plasticity (Bernhardi et al., 2017) complicates the analysis of brain structural connectivity deterioration due to $\mathrm{AD}$. In the future, the combination of the multimodal MRI techniques (structural, diffusion-weighted, and functional MRI) should yield a comprehensive understanding of the relationship between structural and functional changes during normal aging and $\mathrm{AD}$ progression.

\section{CONCLUSION}

Brain network analysis offers a promising new approach to track and understand aging and AD progression. From this study, we conclude that age-related deterioration in structural networks contributes less to AD patients than healthy old adults. While no significant interaction is identified between aging and $\mathrm{AD}$ propagation in terms of the network-level metrics, significant interaction is found in the parcels of left superior temporal, right pars triangularis, and right precentral in terms of nodal clustering coefficient and betweenness. These findings may explain how network abnormalities in AD patients gradually evolve over time. In summary, our results emphasize age- and AD-related degeneration of specific brain parcels, thus providing novel 
A



Left superior temporal (28)



Right pars triangularis (49)



Right precentral (53)

B
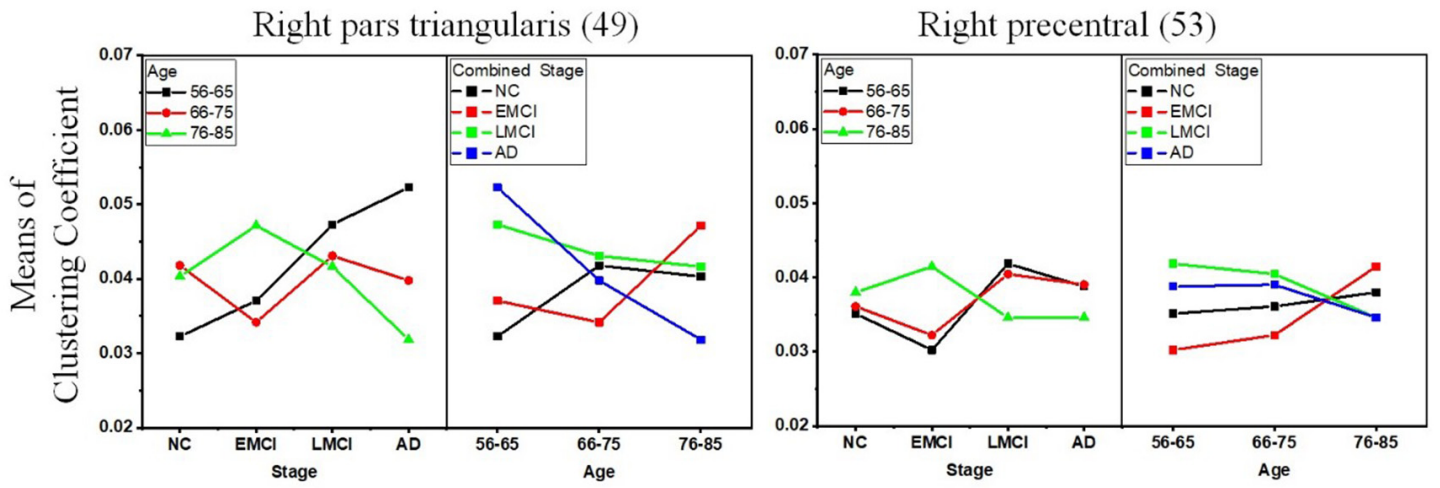

C

Left superior temporal (28)

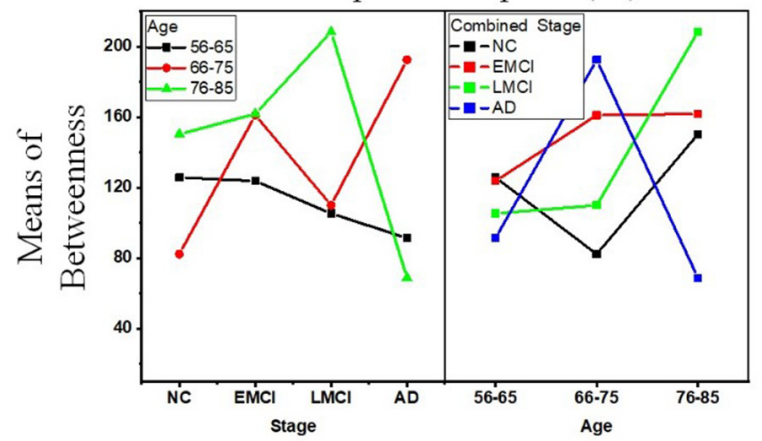

Right pars triangularis (49)



FIGURE 7 | Significant interactions between aging and AD propagation were found in the cortical parcels of left superior temporal, right pars triangularis, and right precentral. (A) Visualization of the parcels of left superior temporal, right pars triangularis, and right precentral. (B) Combined changing trajectories of mean values of clustering coefficient. (C) Combined changing trajectories of mean values of betweenness.

insights into the underlying pathophysiological mechanisms of connectivity alterations over aging and $\mathrm{AD}$ progression. This also indicates the potential of using these parcels' topological metrics as a diagnostic biomarker. Further studies for neurophysiological correlation between aging and $\mathrm{AD}$ progress are still needed to comprehensively assess their cross effects on the integrity of structural connectivity.

\section{DATA AVAILABILITY STATEMENT}

The original contributions presented in the study are included in the article, further inquiries can be directed to the corresponding author.

\section{ETHICS STATEMENT}

Ethical review and approval was not required for the study on human participants in accordance with the local legislation and institutional requirements. Written informed consent for participation was not required for this study in accordance with the national legislation and the institutional requirements.

\section{AUTHOR CONTRIBUTIONS}

ZW analyzed and interpreted the data and was a major contributor in writing the manuscript. YG assisted in analyzing data and interpreting the results, and also contributed to 
manuscript writing. TP assisted in interpretation of the results and manuscript writing. JB performed the statistical analysis and assisted in manuscript writing. JS assisted in analyzing and interpreting the data, and also contributed to manuscript writing. PS assisted in the result interpretation and manuscript writing and revision. YZ supervised the design of study, interpreted results, and contributed to manuscript writing. All authors contributed to the article and approved the submitted version.

\section{FUNDING}

The research was supported in part by Natural Science Foundation of Zhejiang Province (LY20E070005, LY17E070007), National Natural Science Foundation of China (51207038), China Scholarship Council, and the University of Houston. Data collection and sharing for this project was funded by the Alzheimer's Disease Neuroimaging Initiative (ADNI; National Institutes of Health Grant U01 AG024904) and DOD ADNI (Department of Defense award number W81XWH12-2-0012). ADNI is funded by the National Institute on Aging, the National Institute of Biomedical Imaging and Bioengineering, and through generous contributions from the

\section{REFERENCES}

Aeby, A., De Tiege, X., Creuzil, M., David, P., Baleriaux, D., Van Overmeire, B., et al. (2013). Language development at 2 years is correlated to brain microstructure in the left superior temporal gyrus at term equivalent age: a diffusion tensor imaging study. NeuroImage 78, 145-151. doi: 10.1016/j. neuroimage.2013.03.076

Aisen, P. S., Petersen, R. C., Donohue, M. C., Gamst, A., Raman, R., Thomas, R. G., et al. (2010). Clinical core of the Alzheimer's disease neuroimaging initiative: progress and plans. Alzheimers Dement. 6, 239-246. doi: 10.1016/j.jalz.2010. 03.006

Bassett, D. S., Brown, J. A., Deshpande, V., Carlson, J. M., and Grafton, S. T. (2011). Conserved and variable architecture of human white matter connectivity. NeuroImage 54, 1262-1279. doi: 10.1016/j.neuroimage.2010. 09.006

Bell-McGinty, S., Lopez, O. L., Meltzer, C. C., Scanlon, J. M., Whyte, E. M., Dekosky, S. T., et al. (2005). Differential cortical atrophy in subgroups of mild cognitive impairment. Arch. Neurol. 62, 1393-1397. doi: 10.1001/archneur.62. 9.1393

Bernhardi, R., Bernhardi, L. E., and Eugenin, J. (2017). What is neural plasticity? Adv. Exp. Med. Biol. 1015, 1-15. doi: 10.1007/978-3-319-62 817-2_1

Betzel, R. F., Byrge, L., He, Y., Goni, J., Zuo, X., and Sporns, O. (2014). Changes in structural and functional connectivity among resting-state networks across the human lifespan. NeuroImage 102, 345-357. doi: 10.1016/j.neuroimage.2014. 07.067

Burzynska, A. Z., Preuschhof, C., Bäckman, L., Nyberg, L., Li, S.-C., Lindenberger, U., et al. (2010). Age-related differences in white matter microstructure: region-specific patterns of diffusivity. NeuroImage 49, 2104-2112. doi: 10.1016/j.neuroimage.2009.09.041

Cao, Q., Shu, N., An, L., Wang, P., Sun, L., Xia, M., et al. (2013). Probabilistic diffusion tractography and graph theory analysis reveal abnormal white matter structural connectivity networks in drug-naive boys with attention deficit/hyperactivity disorder. J. Neurosci. 33, 10676-10687. doi: 10.1523/JNEUROSCI.4793-12.2013

Dai, Z., Lin, Q., Li, T., Wang, X., Yuan, H., Yu, X., et al. (2019). Disrupted structural and functional brain networks in Alzheimer's disease. Neurobiol. Aging 75, 71-82. doi: 10.1016/j.neurobiolaging.2018.11.005 following: AbbVie, Alzheimer's Association; Alzheimer's Drug Discovery Foundation; Araclon Biotech; BioClinica, Inc.; Biogen; Bristol-Myers Squibb Company; CereSpir, Inc.; Cogstate; Eisai Inc.; Elan Pharmaceuticals, Inc.; Eli Lilly and Company; EuroImmun; F. Hoffmann-La Roche Ltd and its affiliated company Genentech, Inc.; Fujirebio; GE Healthcare; IXICO Ltd.; Janssen Alzheimer Immunotherapy Research and Development, LLC.; Johnson and Johnson Pharmaceutical Research and Development LLC.; Lumosity; Lundbeck; Merck and Co., Inc.; Meso Scale Diagnostics, LLC.; NeuroRx Research; Neurotrack Technologies; Novartis Pharmaceuticals Corporation; Pfizer Inc.; Piramal Imaging; Servier; Takeda Pharmaceutical Company; and Transition Therapeutics. The Canadian Institutes of Health Research is providing funds to support ADNI clinical sites in Canada. Private sector contributions are facilitated by the Foundation for the National Institutes of Health ${ }^{4}$. The grantee organization is the Northern California Institute for Research and Education, and the study is coordinated by the Alzheimer's Therapeutic Research Institute at the University of Southern California. ADNI data are disseminated by the Laboratory for Neuro Imaging at the University of Southern California.

$4_{\text {www.fnih.org }}$

Daianu, M., Jahanshad, N., Nir, T. M., Jack, C. R. Jr., Weiner, M. W. Bernstein, M. A., et al. (2015). Rich club analysis in the Alzheimer's disease connectome reveals a relatively undisturbed structural core network. Hum. Brain Mapp. 36, 3087-3103. doi: 10.1002/hbm.22830

Daianu, M., Jahanshad, N., Nir, T. M., Toga, A. W., Jack, C. R. Jr., Weiner, M. W., et al. (2013). Breakdown of brain connectivity between normal aging and Alzheimer's disease: a structural k-core network analysis. Brain Connect. 3, 407-422. doi: 10.1089/brain.2012.0137

Damoiseaux, J. S. (2017). Effects of aging on functional and structural brain connectivity. NeuroImage 160, 32-40. doi: 10.1016/j.neuroimage.2017.01.077

delEtoile, J., and Adeli, H. (2017). Graph theory and brain connectivity in Alzheimer's disease. Neuroscientist 23, 616-626. doi: $10.1177 / 1073858417702621$

Fischer, F. U., Wolf, D., Scheurich, A., and Fellgiebel, A. (2015). Altered wholebrain white matter networks in preclinical Alzheimer's disease. Neuroimage Clin. 8, 660-666. doi: 10.1016/j.nicl.2015.06.007

Foundas, A. L., Leonard, C. M., Gilmore, R. L., Fennell, E. B., and Heilman, K. M. (1996). Pars triangularis asymmetry and language dominance. Proc. Natl. Acad. Sci. US A 93, 719-722. doi: 10.1073/pnas.93.2.719

Garcés, P., Pereda, E., Hernández-Tamames, J. A., Del-Pozo, F., Maestú, F., and Pineda-Pardo, J. A. (2016). Multimodal description of whole brain connectivity: a comparison of resting state MEG, fMRI, and DWI. Hum. Brain Mapp. 37, 20-34. doi: 10.1002/hbm.22995

Ghanbari, Y., Smith, A. R., Schultz, R. T., and Verma, R. (2014). Identifying group discriminative and age regressive sub-networks from DTI-based connectivity via a unified framework of non-negative matrix factorization and graph embedding. Med. Image Anal. 18, 1337-1348. doi: 10.1016/j.media.2014. 06.006

Gollo, L. L., Roberts, J. A., Cropley, V. L., Biase, M. A. D., Pantelis, C., Zalesky, A., et al. (2018). Fragility and volatility of structural hubs in the human connectome. Nat. Neurosci. 21, 1107-1116. doi: 10.1038/s41593-018 $-0188-z$

Gong, G., Rosaneto, P., Carbonell, F., Chen, Z. J., He, Y., and Evans, A. C. (2009). Age- and gender-related differences in the cortical anatomical network. J. Neurosci. 29, 15684-15693. doi: 10.1523/JNEUROSCI.2308 $-09.2009$

Guo, C. C., Tan, R., Hodges, J. R., Hu, X., Sami, S., and Hornberger, M. (2016). Network-selective vulnerability of the human cerebellum to 
Alzheimer's disease and frontotemporal dementia. Brain 139, 1527-1538. doi: 10.1093/brain/aww003

Hagmann, P., Cammoun, L., Gigandet, X., Meuli, R., Honey, C. J., Wedeen, V. J., et al. (2008). Mapping the structural core of human cerebral cortex. PLoS Biol. 6:e159. doi: 10.1371/journal.pbio.0060159

He, Y., Chen, Z., and Evans, A. (2008). Structural insights into aberrant topological patterns of large-scale cortical networks in Alzheimer's disease. J. Neurosci. 28, 4756-4766. doi: 10.1523/JNEUROSCI.0141-08.2008

Innocenti, G. M., Dyrby, T. B., Girard, G., Stonge, E., Thiran, J., Daducci, A., et al. (2019). Topological principles and developmental algorithms might refine diffusion tractography. Brain Struct. Funct. 224, 1-8. doi: 10.1007/s00429-0181759-1

Jack, C. R. Jr., Bernstein, M. A., Fox, N. C., Thompson, P., Alexander, G., Harvey, D., et al. (2008). The Alzheimer's disease neuroimaging initiative (ADNI): MRI methods. J. Magn. Reson. Imaging 27, 685-691. doi: 10.1002/jmri. 21049

Li, R., Rui, G., Zhao, C., Wang, C., Fang, F., and Zhang, Y. (2020). Functional network alterations in patients with amnestic mild cognitive impairment characterized using functional near-infrared spectroscopy. IEEE Trans. Neural Syst. Rehabil. Eng. 28, 123-132. doi: 10.1109/TNSRE.2019. 2956464

Lo, C.-Y., Wang, P.-N., Chou, K.-H., Wang, J., He, Y., and Lin, C.-P. (2010). Diffusion tensor tractography reveals abnormal topological organization in structural cortical networks in Alzheimer's disease. J. Neurosci. 30, 16876-16885. doi: 10.1523/JNEUROSCI.4136-10.2010

Maier-Hein, K. H., Neher, P. F., Houde, J., Côté, M., Garyfallidis, E., Zhong, J., et al. (2017). The challenge of mapping the human connectome based on diffusion tractography. Nat. Commun. 8:1349. doi: 10.1038/s41467-017 $-01285-\mathrm{x}$

Merio, J. G. (2019). White matter hyperintensities on magnetic resonance imaging: what is a clinician to do? Mayo Clin. Proc. 94, 380-382. doi: 10.1016/j.mayocp. 2019.01.016

Meunier, D., Achard, S., Morcom, A., and Bullmore, E. (2009). Age-related changes in modular organization of human brain functional networks. NeuroImage 44, 715-723. doi: 10.1016/j.neuroimage.2008.09.062

Morabito, F. C., Campolo, M., Labate, D., Morabito, G., Bonanno, L., Bramanti, A., et al. (2015). A longitudinal EEG study of Alzheimer's disease progression based on a complex network approach. Int. J. Neural Syst. 25:1550005. doi: 10.1142/S0129065715500057

Nir, T. M., Villalon-Reina, J. E., Prasad, G., Jahanshad, N., Joshi, S. H., Toga, A. W., et al. (2015). Diffusion weighted imaging-based maximum density path analysis and classification of Alzheimer's disease. Neurobiol. Aging 36, S132-S140. doi: 10.1016/j.neurobiolaging.2014.05.037

Ossenkoppele, R., Lyoo, C. H., Sudre, C. H., van Westen, D., Cho, H., Ryu, Y. H., et al. (2020). Distinct tau PET patterns in atrophy-defined subtypes of Alzheimer's disease. Alzheimers Dement. 16, 335-344. doi: 10.1016/j.jalz.2019. 08.201

Otte, W. M., Van Diessen, E., Paul, S., Ramaswamy, R., Rallabandi, V. P. S., Stam, C. J., et al. (2015). Aging alterations in whole-brain networks during adulthood mapped with the minimum spanning tree indices: the interplay of density, connectivity cost and life-time trajectory. NeuroImage 109, 171-189. doi: 10.1016/j.neuroimage.2015.01.011

Palop, J. J., Chin, J., and Mucke, L. (2006). A network dysfunction perspective on neurodegenerative diseases. Nature 443, 768-773. doi: 10.1038/nature05289

Perl, D. P. (2010). Neuropathology of Alzheimer's disease. Mt. Sinai J. Med. 77, 32-42. doi: $10.1002 / \mathrm{msj} .20157$

Peters, A. (2002). The effects of normal aging on myelin and nerve fibers: a review. J. Neurocytol. 31, 581-593. doi: 10.1023/a:1025731309829

Petersen, R. C., Aisen, P. S., Beckett, L. A., Donohue, M. C., Gamst, A. C., Harvey, D. J., et al. (2010). Alzheimer's disease neuroimaging initiative (ADNI) clinical characterization. Neurology 74, 201-209. doi: 10.1212/WNL. 0b013e3181cb3e25

Potvin, O., Dieumegarde, L., and Duchesne, S. (2017). Freesurfer cortical normative data for adults using Desikan-Killiany-Tourville and ex vivo protocols. NeuroImage 156, 43-64. doi: 10.1016/j.neuroimage.2017. 04.035

Raj, A., LoCastro, E., Kuceyeski, A., Tosun, D., Relkin, N., and Weiner, M. (2015). Network diffusion model of progression predicts longitudinal patterns of atrophy and metabolism in Alzheimer's disease. Cell Rep. 10, 359-369. doi: 10.1016/j.celrep.2014.12.034

Reishofer, G., Studencnik, F., Koschutnig, K., Deutschmann, H., Ahammer, H., and Wood, G. (2018). Age is reflected in the fractal dimensionality of MRI diffusion based tractography. Sci. Rep. 8:5431. doi: 10.1038/s41598-018 $-23769-6$

Risacher, S. L., Saykin, A. J., Wes, J. D., Shen, L., Firpi, H. A., and McDonald, B. C. (2009). Baseline MRI predictors of conversion from MCI to probable $\mathrm{AD}$ in the $\mathrm{ADNI}$ cohort. Curr. Alzheimer Res. 6, 347-361. doi: $10.2174 / 156720509788929273$

Rubinov, M., and Sporns, O. (2010). Complex network measures of brain connectivity: uses and interpretations. NeuroImage 52, 1059-1069. doi: 10.1016/j.neuroimage.2009.10.003

Sheffield, J. M., Rogers, B. P., Blackford, J. U., Heckers, S., and Woodward, N. D. (2019). Accelerated aging of functional brain networks supporting cognitive function in psychotic disorders. Biol. Psychiatry 86, 240-248. doi: 10.1016/j. biopsych.2018.12.016

Sinke, M. R. T., Otte, W. M., Christiaens, D., Schmitt, O., Leemans, A van der Toorn, A., et al. (2018). Diffusion MRI-based cortical connectome reconstruction: dependency on tractography procedures and neuroanatomical characteristics. Brain Struct. Funct. 223, 2269-2285. doi: 10.1007/s00429-018$1628-y$

Smith, R. E., Tournier, J., Calamante, F., and Connelly, A. (2012). Anatomicallyconstrained tractography: improved diffusion MRI streamlines tractography through effective use of anatomical information. NeuroImage 62, 1924-1938. doi: 10.1016/j.neuroimage.2012.06.005

Sotiropoulos, S. N., and Zalesky, A. (2019). Building connectomes using diffusion MRI: why, how and but. NMR Biomed. 32:e3752. doi: 10.1002/nbm.3752

Stawarczyk, D., Grandjean, J., Salmon, E., and Collette, F. (2012). Perceptual and motor inhibitory abilities in normal aging and Alzheimer disease (AD): a preliminary study. Arch. Gerontol. Geriat. 54, 152-161. doi: 10.1016/j.archger. 2011.12.004

Teipel, S., Grothe, M. J., Zhou, J., Sepulcre, J., Dyrba, M., and Sorg, C. (2016) Measuring cortical connectivity in Alzheimer's disease as a brain neural network pathology: toward clinical applications. J. Int. Neuropsychol. Soc. 22 138-163. doi: 10.1017/S1355617715000995

Tong, Q., He, H., Gong, T., Li, C., Liang, P., Qian, T., et al. (2019). Reproducibility of multi-shell diffusion tractography on traveling subjects: a multicenter study prospective. Magn. Reson. Imaging 59, 1-9. doi: 10.1016/j.mri.2019. 02.011

Tong, Q., He, H., Gong, T., Li, C., Liang, P., Qian, T., et al. (2020). Multicenter dataset of multi-shell diffusion MRI in healthy traveling adults with identical settings. Sci. Data 7:157. doi: 10.1038/s41597-020-0493-8

Tournier, J.-D., Yeh, C.-H., Calamante, F., Cho, K.-H., Connelly, A., and Lin, C. P. (2008). Resolving crossing fibres using constrained spherical deconvolution: validation using diffusion-weighted imaging phantom data. NeuroImage 42 617-625. doi: 10.1016/j.neuroimage.2008.05.002

Tuch, D. S., Reese, T. G., Wiegell, M. R., and Wedeen, V. J. (2003). Diffusion MRI of complex neural architecture. Neuron 40, 885-895. doi: 10.1016/s08966273(03)00758-x

Tzourio-Mazoyer, N., Landeau, B., Papathanassiou, D., Crivello, F., Etard, O., Delcroix, N., et al. (2002). Automated anatomical labeling of activations in SPM using a macroscopic anatomical parcellation of the MNI MRI single-subject brain. NeuroImage 15, 273-289. doi: 10.1006/nimg.20 01.0978

van den Heuvel, M. P., and Sporns, O. (2013). Network hubs in the human brain. Trends Cogn. Sci. 17, 683-696. doi: 10.1016/j.tics.2013.09.012

Voevodskaya, O., Pereira, J. B., Volpe, G., Lindberg, O., Stomrud, E., Van Westen, D., et al. (2018). Altered structural network organization in cognitively normal individuals with amyloid pathology. Neurobiol. Aging 64, 15-24. doi: 10.1016/j.neurobiolaging.2017.11.014

Wu, Z., Peng, Y., Selvaraj, S., Schulz, P. E., and Zhang, Y. (2020). Development of brain structural network over age 8: a diffusion weighted imaging study using ensemble average propagator. Front. Aging Neurosci. 12:61. doi: 10.3389/fnagi. 2020.00061

Wu, Z., Xu, D., Potter, T., and Zhang, Y. (2019). Effects of brain parcellation on the characterization of topological deterioration in Alzheimer's disease. Front. Aging Neurosci. 11:113. doi: 10.3389/fnagi.2019.00113 
Yao, Z., Zhang, Y., Lin, L., Zhou, Y., Xu, C., and Jiang, T. (2010). Abnormal cortical networks in mild cognitive impairment and Alzheimer's disease. PLoS Comput. Biol. 6:e1001006. doi: 10.1371/journal.pcbi.1001006

Yousry, T. A., Schmid, U. D., Alkadhi, H., Schmidt, D., Peraud, A., Buettner, A., et al. (1997). Localization of the motor hand area to a knob on the precentral gyrus. A new landmark. Brain 120, 141-157. doi: 10.1093/brain/ 120.1.141

Zhao, T., Cao, M., Niu, H., Zuo, X.-N., Evans, A., He, Y., et al. (2015). Agerelated changes in the topological organization of the white matter structural connectome across the human lifespan. Hum. Brain Mapp. 36, 3777-3792. doi: $10.1002 / \mathrm{hbm} .22877$
Conflict of Interest: The authors declare that the research was conducted in the absence of any commercial or financial relationships that could be construed as a potential conflict of interest.

Copyright (C) 2021 Wu, Gao, Potter, Benoit, Shen, Schulz, Zhang and The Alzheimer's Disease Neuroimaging Initiative. This is an open-access article distributed under the terms of the Creative Commons Attribution License (CC BY). The use, distribution or reproduction in other forums is permitted, provided the original author(s) and the copyright owner(s) are credited and that the original publication in this journal is cited, in accordance with accepted academic practice. No use, distribution or reproduction is permitted which does not comply with these terms. 